\title{
PROPUESTA CURRICULAR ALTERNATIVA PARA EL EJERCICIO $Y$ LA VIVENCIA DE LOS DERECHOS HUMANOS EN LAS INSTITUCIONES EDUCATIVAS
}

\author{
Alternative curricular proposal for the practice and experience \\ of Human Rights in educational institutions
}

Nelson Ernesto López Jiménez
María Fernanda Pérez Gutiérrez
**

López, N.E., Pérez, M.F. \& Perdomo, W.R. (2016) Propuesta curricular alternativa para el ejercicio y la vivencia de los derechos humanos en las instituciones educativas. Revista PACA, 8, 115-138

\section{Resumen}

Este artículo tiene como propósito central analizar las expresiones del currículo oculto en algunas instituciones educativas del Departamento del Huila y sus implicaciones en las distintas formas de violencia escolar en el contexto de la política sobre los Derechos Humanos que existe en Colombia.

La problemática de los Derechos Humanos en América Latina y en Colombia ha adquirido una relevancia fundamental debido a la existencia

\footnotetext{
*Doctor en Educación. Docente de tiempo completo de la Universidad Surcolombiana, Director del grupo de investigación PACA. Email: nelopez53@gmail.com.

${ }^{* *}$ Magíster en Educación. Co-investigadora del grupo de investigación PACA.

Email: nanita_12_88@hotmail.com.

${ }^{* * *}$ Comunicador social y periodista. Co-investigador del grupo de investigación PACA.

Email:wilsonperdomo07@gmail.com.
} 
de condiciones reales que afirman y denuncian su violación sistemática, producto de políticas y acciones fundamentadas en las formas de violencias y en el autoritarismo de muchos de los gobiernos de los países del área. En este contexto resulta importante indagar sobre la responsabilidad que tienen las Instituciones Educativas IE en cuanto a la defensa, el reconocimiento y el respeto de los Derechos Humanos DDHH. ${ }^{23}$

Se plantea que a través del currículo oculto se vehiculizan una serie de violencias, que al no ser consideradas relevantes, crean el espacio propicio para la violación permanente de los DDHH en la medida que no se acepta ni respeta la diferencia, la otredad, la singularidad, creando un ambiente de atropello, abuso, inseguridad, incertidumbre, miedo, que afecta y pone en cuestión los logros señalados en los diferentes propósitos y principios de las IE.

En síntesis, la problemática que orienta y determina este artículo se concreta en el estudio, análisis, caracterización e intervención de los Derechos Humanos y sus implicaciones con las diversas formas de violencia escolar expresadas en el currículo oculto.

Palabras clave: Derechos Humanos, currículo oculto, violencia escolar, Educación, Currículo.

\section{Abstract}

The main purpose of this article is to analyze the expressions of the hidden curriculum in some educational institutions of the department of Huila and their implications in the different forms of school violence in the context of the policy on Human Rights that exists in Colombia.

The concern about Human Rights in Latin America and Colombia has acquired a fundamental importance due to the existence of real conditions that affirm and denunciate its systematic violation, product of policies and actions based on the forms of violence and the authoritarianism of many governments of the countries in the area. In this context, it is important

\footnotetext{
${ }^{23}$ Esta es una de las conclusiones producto de la primera fase de esta investigación que se orientó a sustentar la relación tensionante y conflictiva entre Currículo y los DDHH.
} 
to ask about the responsibility of the IE (Educational Institutions) for the defense, recognition and respect of Human Rights.

It is proposed that through the hidden curriculum a series of violence are transmitted, that, when not considered relevant, create the conducive space for the permanent violation of Human Rights insofar as the difference, the otherness, and the singularity are not accepted or respected, creating an environment of abuse, insecurity, uncertainty, fear, which affects and calls into question the achievements indicated in the different purposes and principles of IE (Educational Institutions).

In summary, the problem that guides and determines this article is concretized in the study, analysis, characterization and intervention of the Human Rights and its implications for the different forms of school violence expressed in the hidden curriculum.

Key words: Human Rights, hidden curriculum, school violence, Education, Curriculum.

\section{Introducción}

El Grupo de Investigación PACA, Categoría A de Colciencias, viene trabajando de manera sistemática las problemáticas surgidas de la relación existente entre los Derechos Humanos y las diferentes realidades curriculares presentes en los procesos formativos.

El currículo se asume como el proceso de selección, organización y distribución del conocimiento considerado válido para alcanzar los propósitos de formación ${ }^{24}$ (López: 2003), de ahí, que se considere importante indagar de manera puntual sobre los impactos y efectos de lo que se ha denominado "currículo oculto", es decir, lo que no se dice en los documentos oficiales institucionales que constituyen el Discurso Regulativo Institucional DRI ${ }^{25}$ (PEI, Estatuto General, Manual de Convivencia, otros) pero que existe, se evidencia, se concreta, se presenta en todas y cada

\footnotetext{
${ }^{24}$ López Jiménez Nelson E. (2003) La De-Construcción Curricular. Editorial Magisterio.

${ }^{25}$ Díaz V. y López, N. E. (2001). El Discurso Pedagógico Oficial y la Educación Superior en Colombia. Colciencias, Universidad Surcolombiana.
} 
una de las interacciones que los miembros de las instituciones educativas IE desarrollan en el marco del proceso formativo. ${ }^{26}$

El presente artículo se justifica de manera concreta debido a la preocupación de hacer inmersión en la realidad de las IE (que integra universidades, instituciones de educación superior, colegios, escuelas, centros de educación no formal, otras), para identificar, estudiar, caracterizar, intervenir y superar las diferentes formas de violencia(s), desconocimiento, violación, restricción, negación de los derechos humanos que le asisten a todo ser humano, por su condición.

El currículo oculto "lo que realmente es válido y real en las IE" no ha sido asumido como problemática sustantiva, de ahí que cuando se analiza la realidad escolar, es muy común encontrar información relacionada con su dimensión cuantitativa (número de alumnos, número de aulas, profesor de planta, ocasionales, medio tiempo, cátedra, índices de permanencia y abandono, graduados, etc), la dimensión cualitativa (que explicaría la razón de ser de los procesos) no ha sido abordada de manera consistente, seria y sistemática, razón por la cual, esta investigación pretende, de manera concreta, profundizar en la "naturaleza de las relaciones sociales que se dan en la IE y a través de ellas determinar las diferentes formas de violencia(s) que ellas agencian", lo cual permite contar con referentes básicos y determinantes para la construcción de identidad y subjetividad de estudiantes, profesores y directivos.

\section{Referentes teóricos, conceptuales y legales}

Uno de los referentes que se retomaron para esta investigación es el planteamiento teórico del currículo oculto y su relación con la pertinencia de los Derechos Humanos desde las políticas públicas.

Se considera adecuado resaltar el carácter polisémico que comporta la noción de currículo. Existen diferentes planteamientos en torno a la concepción y representación del mismo (Castoriadis: 2004); lo que hay que destacar en cualquier definición de currículo son los aspectos o

\footnotetext{
${ }^{26}$ Según Jurgo Torres (1994) "la investigación etnográfica nos permite ver con mayor claridad lo que realmente sucede en el interior de ese nicho ecológico que son las instituciones educativas IE. Currículo Oculto. Ediciones MORATA
} 
intereses que se pretenden enfatizar en cada una de ellos; existen concepciones que están íntimamente relacionadas con la transmisión de conocimientos y su carácter o intencionalidad están supeditados a los conocimientos o contenidos que transmiten. Por otra parte, se dan concepciones sustentadas en la crítica y transformación de la realidad y contextos en donde se desarrolla el proceso curricular, que se adentran en las problemáticas que sustentan la razón de ser del mismo (Apple, Giroux, Magendzo, Ferrada, Stenhouse, Díaz, López Jiménez, entre otros.).

Dado que el propósito central de la presente investigación es el de determinar la relación existente entre el currículo oculto, los DDHH y las formas de violencia (s) que se generan en la IE, se considera conveniente explicitar la concepción que se maneja de currículo. Este se asume como un "proceso eminentemente investigativo, al cual se accede por aproximaciones sucesivas y resultado de un trabajo de elaboración permanente y colectiva donde la indagación sistemática, la pregunta, la duda, el interrogante y la incertidumbre se convierten en elementos dinamizadores de esa búsqueda"(López:2003).

No obstante, se observa una problemática relacionada con la coherencia y consistencia entre el diseño, el desarrollo y la evaluación curricular; es evidente su divorcio y separación, lo cual genera un distanciamiento entre lo que se dice (planea) y lo que se desarrolla (ejecuta), y es en esta brecha o distancia que se expresa el currículo oculto. ${ }^{27}$

Se trata de explicar inicialmente la fractura existente entre la escuela y la realidad implícita (currículo oculto) y sus implicaciones con el ejercicio de los DDHH. En esta concepción se descarta de manera contundente la pretensión de "neutralidad valorativa" que acompaña buena parte de las elaboraciones curriculares del denominado currículo enciclopédico, academicista y asignaturista (currículo explícito). Es conveniente indicar que el proceso curricular adquiere una naturaleza política dado que comporta estructuras de poder y control (Bernstein: 2003) que a su vez agencian determinaciones en cuanto a la construcción de subjetividad y

\footnotetext{
${ }^{27}$ López Jiménez, Nelson. (2006). Retos para la Construcción Curricular. Colección MESA REDONDA, No. 42. Editorial Magisterio. Tercera Edición
} 
de identidad. En este contexto es importante destacar el llamado que nos hace Gentili (2015) "la fragmentación y segmentación del sistema escolar, en particular de la oferta de nivel medio; el desprecio o la indiferencia hacia los grupos más postergados, por ejemplo los sectores rurales, la desigualdad y la injusticia social que han interferido en las condiciones efectivas para hacer realidad demandas de justicia e igualdad inherentes a cualquier democracia moderna; la relevancia de una parafernalia de leyes, tratados $y$ acuerdos nacionales e internacionales destinados a proteger $y$ promover los Derechos Humanos y, dentro de estos, de forma indivisible, la educación, en un marco sorprendente de ineficacia de la ley y de los recursos jurídicos que deberían hacerla cumplir."

El currículo oculto "hace referencia a todos aquellos conocimientos, destrezas, actitudes y valores que se adquieren mediante la participación en procesos de enseñanza y aprendizaje $y$, en general, en todas las interacciones que suceden día a día en las aulas y centros de enseñanza. Estas adquisiciones, sin embargo, nunca llegan a explicitarse como metas educativas a lograr de manera intencional". ${ }^{28}$

El currículo oculto se concibe como "lo que realmente se produce en las IE como consecuencia de las interacciones sociales de los diferentes actores de la realidad educativa y que no está explicitado en el Discurso Regulativo Institucional DRI" 29. El análisis desarrollado demuestra que las formas de violencia(s) que se presentan en la IE son agenciadas, desarrolladas y viabilizadas por el currículo oculto, creando todas las condiciones para el desconocimiento, violación, conculcación de los DDHH. En esta perspectiva resulta muy útil consignar que "el reconocimiento de la educación como derecho humano fundamental, desde la Declaración de 1948, constituye el punto de partida para la construcción de toda política educativa que aspire a sustentar los fundamentos de una democracia efectiva y duradera. Así, el cumplimiento de los principios que fundamentan el carácter de la educación como derecho humano en dicha proclama y en la Convención sobre los Derechos del Niño resulta una obligación jurídica y ética por parte de los 192 Estados miembros de las Naciones Unidas. Violar este derecho

\footnotetext{
${ }^{28}$ Torres, S. Jurgo. (1994). "El Curriculum Oculto". Ediciones MORATA. Quinta Edición. Madrid.

${ }^{29}$ López y Díaz. (2004). El discurso Pedagógico Oficial y la Educación Superior en Colombia. ColcienciasUniversidad Surcolombiana.
} 
significa violar un Derecho Humano Fundamental y, de esta forma, poner en cuestionamiento el orden jurídico sobre el cual se sustentan los principios de una sociedad libre e igualitaria, solidaria y democrática. Si todo esto es correcto, deberíamos asumir sin medias palabras que negar a un pueblo el derecho a la educación constituye un delito contra la humanidad" (Gentili: 2015).

El equipo investigador consideró necesario realizar una revisión sistemática del Decreto Único Reglamentario del Sector Educativo Colombiano, 1075 del 26 de mayo de 2015 del Ministerio de Educación Nacional, con el propósito de identificar los aspectos específicos que en materia de prevención, regulación y defensa de los Derechos Humanos en la escuela se encuentran consignados en dicha norma.

La importancia del análisis de la dimensión legal y jurídica del campo donde se ubica la problemática de los DDHH intenta responder a una postura relacionada con la insuficiencia de las normas si estas se quedan solamente en el campo enunciativo. Su validez, pertinencia y aplicabilidad exigen una relación constructiva entre las dimensiones teóricas y prácticas de su aplicación; no obstante, es necesario precisar, como a continuación se reseña, que no es por falta de regulaciones en el orden jurídico que los DDHH no se ejercen ni se respetan en la IE .

El Discurso Regulativo General DRG (Políticas Públicas en Colombia) contempla de manera detenida y puntual las diferentes regulaciones cuyo cumplimiento garantizaría el ejercicio de los DDHH en la IE. No obstante, se demostrará más adelante como resultado del trabajo de campo y su respectivo análisis, que la normatividad sobre las conductas y comportamientos que garantizan el ejercicio de los DDHH en la IE no son suficientes ni se convierten en prueba de su respeto; por el contrario, se advierte con preocupación que en muchas realidades escolares, no superan el nivel discursivo de las mismas. Esto significa que no es que no existan normas, lo preocupante es que no se cumplen.

En relación con la metodología utilizada es conveniente señalar que la investigación se orientó desde la perspectiva cualitativa que implica la utilización y recogida de una gran variedad de materiales-entrevista, experiencia personal, historias de vida, observaciones, textos históricos, 
imágenes, sonidos - que describen la rutina y las situaciones problemáticas y los significados en la vida de las personas". (Rodríguez, Gil y otros, 2011).

Se logró que la "voz de los actores" (estudiantes, profesores, directivos, padres de familia) encontraran un escenario propicio para establecer diálogos intencionados en torno a lo que realmente acontece en el proceso formativo. Se analizaron las miradas, concepciones, percepciones que los protagonistas de la realidad escolar han construido y esperan construir cuando abordan la problemática del ejercicio de los DDHH en la IE. "EI sentido está abierto a la subjetividad, por lo tanto la atención a la interioridad es muy grande, central, de otra manera el sujeto quedaría ciego a sus impulsos, emociones, sentimientos y recuerdos". ${ }^{30}$

El trabajo de campo permitió la obtención de diferentes puntos de vista de actores de la realidad escolar, concretamente los rectores de IE, coordinadores académicos y orientadores de IE, profesores de instituciones educativas de Educación Inicial, Básica y Media; estudiantes de Maestría en Educación y estudiantes de las IE.

El trabajo de campo permitió el desarrollo de entrevistas, diplomados, foros virtuales y la elaboración de un video testimonial que aportaron a la investigación elementos fundamentales para el logro de los propósitos perseguidos.

\section{¿Qué se obtuvo de la investigación realizada ?}

Según los hallazgos obtenidos en el análisis de las prácticas e interacciones sociales establecidas en las IE del Huila participantes en esta investigación, se comprobó la existencia de acciones de violencia de género que se caracterizan por la marginación, burla y sometimiento de las personas por su condición e identidad sexual y/o de género.

La comunidad académica señaló que la violencia de género se evidencia en los espacios propios del currículo oculto y que los principales

\footnotetext{
${ }^{30}$ Galindo, Cáceres. (1995) Apuntes de metodología en investigación cualitativa. Programa Cultura, Centro Universitario de Investigaciones Sociales. Universidad de Colima México.
} 
protagonistas son los estudiantes; indican que estos ejercen violencia a través de la discriminación, el señalamiento, el acoso y las agresiones a sus compañeros que no están dentro de los estereotipos de género establecidos socialmente.

Los rectores y coordinadores afirmaron que las manifestaciones de violencia de género entre los estudiantes se originan en las prácticas sexistas y homofóbicas que se viven en los contextos familiares, adoptadas y reproducidas por los niños, niñas y jóvenes. Es así como el machismo habita en las IE, teniendo en cuenta que se reproducen y asignan roles socialmente determinados según el género.

De otra parte, la comunidad educativa indicó que en las IE habita una forma de violencia silenciosa e invisible que pasa desapercibida denominada "violencia simbólica". Según las audiencias consultadas, esta forma de violencia es ejercida por los docentes hacia sus estudiantes a través de manifestaciones como el tono de voz, señalamientos, gestos $y$, en ocasiones, ignorando al estudiante cuando desea participar en los diferentes procesos pedagógicos.

Los rectores, coordinadores, orientadores y docentes consideran que la violencia simbólica es difícil de percibir, intervenir y solucionar, teniendo en cuenta que las prácticas e interacciones comunicativas en la escuela tradicionalmente han sido mediadas por relaciones de poder y control autoritarias y jerárquicas ${ }^{31}$ ejercidas por el maestro y naturalizada por el estudiante.

Es importante mencionar que la comunidad educativa considera que los espacios virtuales tienen aspectos positivos porque hay diversas oportunidades de lograr aprendizajes significativos en estudiantes, facilitan la socialización y desarrollan positivamente habilidades; sin embargo, también se presentan aspectos negativos asociados al uso de redes sociales e internet porque puede haber ruptura de relaciones sociales y generación de conflictos.

${ }^{31}$ Bernstein, Basil. (1990).Construcción Social del Discurso Pedagógico. Corprodic. Bogotá. 
Según los coordinadores, estudiantes y orientadores escolares en sus IE se presenta violencia cibernética promovida y ejercida por los estudiantes a través de diferentes redes sociales como Facebook y Whatsapp, realizando comentarios ofensivos, publicando videos e imágenes, exponiendo aspectos privados de una persona para agredir y atentar contra la integridad de sus pares.

Los agentes educativos señalaron que se presenta discriminación y es considerada como el trato diferenciado que se da a otra persona por su procedencia cultural y étnica y/o por sus creencias religiosas. Es pertinente indicar que la comunidad educativa que participó en el desarrollo de esta investigación considera que estas formas de violencias es producto de un modelo social inequitativo, excluyente y homogeneizador, donde las singularidades son discriminadas socialmente.

La comunidad educativa expresó que en las IE existe un Manual de Convivencia que consagra reglas institucionales relacionadas con el comportamiento y la presentación personal que promueve la violencia, teniendo en cuenta que homogeniza modelos y estereotipos sociales y coarta la diversidad, tradiciones, costumbres e identidad cultural de la comunidad educativa.

En cuanto a la educación religiosa en los establecimientos educativos, la normatividad $^{32}$ señala que los estudiantes tienen derecho a la libertad religiosa de optar o no por tomar la educación teológica que ofrece la IE; en tal caso, el colegio debe garantizar la realización de actividades alternativas relacionadas con esta área de acuerdo al Proyecto Educativo Institucional. No obstante, algunas audiencias consultadas indicaron que no se respeta el culto de los niños, niñas y jóvenes en algunas IE del Huila, teniendo en cuenta que los estudiantes son obligados a asistir a las ceremonias católicas porque se carece de docentes y actividades alternativas que atiendan a la población con diferentes cultos.

Es pertinente señalar que la comunidad educativa considera que es necesario que la escuela sea pertinente a las necesidades de sus

\footnotetext{
32 Decreto Único Reglamentario del Sector Educativo Colombiano, 1075 del 26 de mayo de 2015 del Ministerio de Educación Nacional, Artículo 2.3.3.4.4.5.
} 
estudiantes, orientando la formación al contexto que se vive dentro y fuera de esta, con el propósito de promover la igualdad de oportunidades y alternativas para realizar su proyecto de vida, esto enmarcado en la transformación del currículo, las practicas pedagógicas y comunicativas y los manuales de convivencia.

Los estudiantes de las IE señalaron que se presenta una violencia física, entendida como la vulneración del espacio físico de los niños, niñas y jóvenes causada a través de golpes, empujones y jalones.

Manifestaron que las agresiones físicas se dan entre los estudiantes a las afueras de las IE por problemas sentimentales, que en ocasiones traen desde su contexto social y son provocadas por sus propios compañeros. Es indispensable que dentro de las IE se promueva el diálogo como estrategia fundamental para la resolución de los conflictos y la construcción de una nueva escuela para la paz, la equidad y la reconciliación.

\section{Propuesta alternativa para garantizar el ejercicio, respeto, defensa y preservación de los DDHH en las IE}

Teniendo como referente inmediato los resultados obtenidos que hacen alusión concreta a la consideración estructural de asumir los DDHH como una problemática que se concreta en la práctica, en el ejercicio real, no pueden seguir siendo asumidos como contenidos, normas o temáticas que se deben enunciar, para que se conozcan y señalar que existen.

La presente propuesta en materia formativa da cuenta de las problemáticas existentes en la relación DDHH, currículo oculto y violencia(s) y, a partir de las mismas proponer y construir programas y proyectos innovadores que permitan desarrollar estrategias incluyentes, democráticas, participativas, caracterizadas por la pluralidad y la participación, que se identifiquen por ser esencialmente pertinentes con la realidad contextual, cultural, social, política, académica y humana en donde se evidencia y adquiere forma el proceso formativo. En lo que sigue se presentan los aspectos estructurantes de la propuesta curricular alternativa que aluden a sus principios, objetivos, estructura, criterios de implementación y acciones de seguimiento y acompañamiento. 
Principios: Expresan de manera concreta las bases teóricas y conceptuales mediante las cuales se concibe el proceso curricular y que a su vez, determinan la representación, concepción y desarrollo del mismo. Estos son:

Enfoque Investigativo: Al considerar el proceso curricular como un proceso eminentemente investigativo se asume que los problemas, no los temas / contenidos, se convierten en la estrategia central de toda elaboración curricular, lo cual implica un cambio esencial donde se debe superar e intervenir la concepción sobre los DDHH en la actualidad como saberes y conocimientos de los mismos, y abordar la problemática relacionada con el respeto, el ejercicio y el reconocimiento de los DDHH de los niños, niñas y jóvenes que son la esencia de la formación humana, académica, investigativa, cultural, tecnológica que se pretende desarrollar en la IE.

Pertenencia Social: Lo formativo no se agota en lo escolar, es necesario crear estrategias para integrar las lógicas de las otras agencias formativas (los contextos familiar, laboral, artístico, cultural, deportivo, social, entre otros) con las lógicas del contexto escolar. Es una realidad indiscutible que Colombia es un país plurietnico, multicultural y de regiones; la pertenencia social de los procesos curriculares que se adelanten necesariamente debe responder a las necesidades sociales que le han dado origen. No se puede ignorar y desconocer la singularidad, la particularidad de los contextos, a riesgo de homogenizar una realidad que no es homogenizable. Este principio ha servido para cuestionar la validez y legitimidad de las denominadas "pruebas censales" que en Colombia desarrolla el ICFES. ${ }^{33}$

Pertinencia Académica: La pertinencia académica se concibe como "la coherencia existente entre las necesidades educativas y la respuesta académica que se le da a las mismas". La pertinencia académica está asociada a la naturaleza investigativa que se le otorga al currículo, dado que es resultado de una indagación sistemática. De cara a la problemática de la(s) violencia(s) y en relación con la pertinencia académica para

\footnotetext{
${ }^{33}$ López Jiménez Nelson, (2014). "Análisis Crítico de las Evaluaciones Censales". Facultad de Educación, Universidad Surcolombiana.
} 
abordarlas es preciso señalar que "los docentes deberían practicar y enseñar una ética del dialogo entre alumnos que se pelean, dialogo entre profesores y alumnos (...).

¿A estudiado las condiciones biológicas y sociales de la agresividad y propone acertadamente? "transformar la violencia de los alumnos en conflicto, en un conflicto de palabras e ideas, que sí permite el dialogo y por ello mismo se convierte en un aprendizaje para la democracia, la cual necesita conflictos de ideas para no degenerar siempre que tales conflictos ignoren la fuerza física. En consecuencia, sería muy importante encontrar métodos de prevención de la violencia".34

Participación: En el libro sobre La de-construcción curricular ${ }^{35}$, plantea "la participación no es una enunciación retórica, es preciso convertirla en un aval definitivo en y para la de-construcción de una nueva cultura académica. La posibilidad de superar los límites rígidos entre los que planean las estructuras curriculares y los que ejecutan dicha planeación se constituye en una impronta determinante de esta propuesta alternativa (...)".

La participación no necesariamente involucra a los actores de la IE sino que debe abarcar y lograr que otros actores (sociedad civil, organizaciones populares, asociaciones, fundaciones y ciudadanos de a pie), proyecten y realicen programas conjuntos que consoliden la formación lograda en la IE. "No se puede concebir la participación como una enunciación de buena voluntad; por el contrario, las estructuras curriculares surgidas de los procesos participativos comportan la dinámica contradictoria de su formulación, expresan la naturaleza polifónica de su construcción y se erigen como una prueba fehaciente de elaboración por aproximaciones y acuerdos sucesivos" (López J, 2003).

Flexibilidad e Interdisciplinaridad: Este principio está relacionado con la

\footnotetext{
${ }^{34}$ Véanse los trabajos de Éric Debarbieux, director del Observatorio Internacional de la Violencia en la Escuela desde 2004 y los de Élisabeth Maheu que propugna la aplicación de prácticas de no violencia en la escuela. Citado por Morin en su texto "Enseñar a Vivir: manifiesto para cambiar la educación" PAIDOS, 2016.

${ }^{35}$ López Jiménez Nelson.(2003).Colección Seminarium Magisterio. Línea de investigación Curriculum. Editorial Magisterio.
} 
necesidad de reformar nuestro pensamiento, dado que la insularidad, el atomismo, el enciclopedismo han convertido la formación académica en un archipiélago de conocimientos fragmentados, disciplinares y academizados. La interdisciplinaridad se mueve en el contexto de la complejidad que no da opciones a únicas salidas, sino por el contrario advierte la necesidad de integrar y articular diferentes aportes, desarrollos, elaboraciones que aborden la naturaleza compleja de la realidad. El estudio sobre las violencias escolares no puede asumirse desde una óptica disciplinar; por el contrario, la integración de saberes y conocimientos permitirá un abordaje pertinente de dicha problemática "el modo de pensamiento o de conocimiento parcelario, compartimentado, monodisciplinar y cuantificador, conduce a una inteligencia ciega, del mismo modo que la aptitud humana normal para relacionar los conocimientos se sacrifica en aras de la aptitud no menos normal de separarlos $^{136}$. Importante destacar que la prevalencia y la hegemonía de las estructuras curriculares diseñadas por materias o asignaturas separadas, nos hace perder la aptitud de relacionar, la aptitud de contextualizar, lo cual impide avanzar en un proceso de desarrollo curricular con pertenencia social y pertinencia académica.

Integración teoría -práctica: Como se analizó en el marco teórico y conceptual de esta investigación y, concretamente, en la dimensión normativa del sector educación en Colombia (Decreto 1075 de 2015), no basta con la sola enunciación de los posibles procesos a desarrollar; es necesario verificar en la práctica la pertenencia social, la pertinencia académica, la interdisciplinariedad, el enfoque investigativo, creando una alianza inextricable entre lo que se dice y lo que se hace. Como se verá más adelante, la estrategia núcleo temático y problemático $0^{37}$ e convierte en esta propuesta en la estrategia curricular que garantiza una articulación entre la dimensión teórica- práctica del proceso formativo.

En el contexto de la problemática sobre la(s) violencia(s) escolar, y de cara a la realidad curricular hegemónica, se hace necesaria una resignificación en el manejo de los diferentes procesos y acciones de la

\footnotetext{
${ }^{36}$ Morín Edgar "Enseñar a Vivir. Manifiesto para cambiar la educación "PAIDÓS, 2016.

${ }^{37}$ Véase, López Jiménez Nelson (1992) La Reestructuración Curricular de la Educación Superior: Hacia la Integración del Saber. ICFES - Universidad Surcolombiana.
} 
labor formativa. Es necesario debilitar la distancia entre el contexto de formulación curricular y su contexto de realización.

Temporalidad: No existen estructuras curriculares con validez universal; significa lo anterior que si los procesos curriculares responden por los principios de pertenencia social y pertinencia académica, su temporalidad está definida por la naturaleza de las mismas.

Evaluación Permanente: Cuando se habla de evaluación se hace alusión directa a los proceso de valoración y logro de los propósitos perseguidos, razón por la cual se descarta de manera relevante que la evaluación se reduzca a una medición expresada a través de notas o calificaciones. La evaluación inmersa en un contexto investigativo que pretende debilitar las diferentes formas de violencia(s) que se dan en la IE está directamente relacionada con alcanzar la erradicación y debilitamiento de las mismas. ¿Cuál es el NORTE de la propuesta?

Lo que se pretende con esta propuesta, muy relacionada con el ejercicio de los DDHH en la IE, está relacionada con:

- Asumir el proceso curricular como una acción eminentemente investigativa.

- Crear las condiciones mínimas básicas para que los procesos curriculares se caractericen por su pertenencia social y pertinencia académica.

- Contar con argumentos sólidos para defender el ejercicio, el respeto, la defensa y la preservación de los $\mathrm{DDHH}$ en el proceso formativo desarrollado en las IE.

- Contribuir a la creación de una nueva cultura escolar que se integre a la realidad del contexto socioeconómico y cultural del cual hace parte.

- Apoyar y promover la responsabilidad social de la IE como agencia de cambio y transformación de la realidad socio-económica y cultural.

Estructura de la propuesta alternativa para garantizar el ejercicio, respeto y preservación de los DDHH en las IE.

La propuesta de intervención o alternativa para abordar la problemática existente entre el currículo oculto y las formas de violencia(s) que 
obstaculizan el ejercicio, el respeto, la defensa y preservación de los DDHH en la IE, además de ser un objetivo previsto en esta investigación, se convierte en una concepción diferente del proceso de formación, dado que articula los contextos internos y externos donde se expresa y adquiere forma la problemática y que necesariamente se convierte en la arena propicia donde se detectan y priorizan problemas y necesidades que expresan el desconocimiento y la vulneración de los DDHH que les pertenecen a los actores que hacen parte del escenario formativo.

Las necesidades se definen como la distancia existente entre lo que es y lo que debe ser, es decir, entre lo que hay y lo que debe haber de cara a una formación que se orienta fundamentalmente al reconocimiento, el ejercicio, la defensa y la preservación de los DDHH de todos los actores que hacen parte del horizonte formativo; los problemas pueden entenderse como ruidos, irrupciones, rompimientos, vacíos, discontinuidades. Desde esta perspectiva se quiere otorgar una significación diferente y alternativa a los conocimientos, sean estos disciplinarios o interdisciplinarios; se quiere demostrar que el papel que juegan los diferentes conocimientos integrados a las disciplinas no pueden considerarse fines en sí mismos, sino que son medios indispensables para la solución de los problemas y la satisfacción de las necesidades.

En cuanto a las problemáticas y necesidades en la dimensión macro, se hace indispensable señalar de manera clara y contundente que la mayoría de acciones de los países y de las instituciones que de ellos hacen parte, afrontan retos permanentes en relación con las exigencias planteadas por un nuevo contexto caracterizado por la internacionalización de los diversos sectores económicos, culturales, sociales, científicos, tecnológicos, ambientales, que se convierten en evidencias indiscutibles de la existencia de un proceso de globalización que permite precisar que el mundo no es homogéneo toda vez que está constituido de muchas formas culturales, sociales, y aun económicas, que conviven en el mismo tiempo-espacio de la globalización capitalista neoliberal. ${ }^{38}$

\footnotetext{
${ }^{38} \mathrm{Un}$ estudio riguroso sobre la globalización y su impacto o relevancia en la Educación, se encuentra en el libro de Marco Raúl Mejía J. "Educación(es) en la (s) Globalización (es) I y II. Entre el pensamiento único y la nueva crítica". Ediciones desde abajo 2007.
} 
De otra parte, se hace necesario consignar los aspectos relevantes en materia de política pública nacional, que a través de sus variadas y complejas regulaciones señalan el derrotero a seguir en los diferentes campos y sectores, que de manera concreta hacen alusión a las políticas nacionales en torno a la educación. ${ }^{39}$

La confrontación entre las dimensiones macro y micro del contexto en el cual se inserta la educación permitirá definir y determinar las problemáticas o necesidades relacionadas con el ejercicio, el respeto, la defensa y la preservación de los $\mathrm{DDHH}$, que hacen parte de su horizonte de acción y, a partir de ellas, definir las acciones a desarrollar en busca de pertenencia social y pertinencia académica (principios curriculares) de las decisiones y desarrollos a ejecutar.

Una vez identificadas las necesidades y los problemas relacionados con el ejercicio, el respeto, la defensa y la preservación de los DDHH, se avanza en la construcción de una respuesta concreta de cara a los vacíos o debilidades detectados en las diferentes dimensiones en las cuales se expresan las acciones de violencia (étnica, de género, simbólica, física, cibernética, religiosa, socioeconómica, entre otras). Como consecuencia de este desarrollo se podrá definir el Propósito de Intervención estructurado con el fin de debilitar y eliminar las causas y factores que han dado lugar a la(s) violencia(s) encontradas con bases y sustentos de pertenencia social y pertinencia social, académica, cultural, étnica, educativa, que los justifique. ${ }^{40}$

Si se pretende abordar las diferentes causas o factores que generan la(s) violencia(s) es necesario precisar lo que en el contexto de esta propuesta se entiende por formación, concepto que hace alusión concreta a la "simbiosis creativa entre un campo de problemas y un campo de saberes y conocimientos" ${ }^{41}$; significa que los problemas (en este caso expresados

\footnotetext{
${ }^{39}$ En la actualidad en Colombia se discute una nueva Ley de Reforma de la Educación Superior que ha concitado el interés nacional y que ha sido considerada como punto de debate de la comunidad académica nacional.

${ }^{40}$ Los conceptos de pertenencia social y pertinencia académica son categorías analíticas que hacen parte del modelo curricular sustentado por el grupo de investigación PACA. Véase el libro La Deconstrucción curricular. Editorial Magisterio. 2001

${ }^{41}$ Concepción que se convierte en categoría esencial del modelo formativo propuesto por el PACA
} 
a través de las diferentes formas de violencia(s) se abordan con el apoyo y participación de un concierto de saberes y conocimientos, que no necesariamente son académicos (saber popular, ancestral, conocimientos cotidianos, experiencias del mundo de la vida, entre otros); los saberes y conocimientos no estarían desligados de una realidad concreta y singular; por el contrario, se convierten en reacciones y respuestas directas y pertinentes a las problemáticas aludidas. Además, la intervención a las diferentes formas de violencia (s) que se evidencian en la IE, se apoyarán en estructuras curriculares cuya base de sustentación estaría dada por acciones de investigación, asumidas como procesos de indagación sistemáticos permanentes y consolidados a través de las mediaciones o prácticas pedagógicas, cuya esencia concreta se orientará hacia acciones de construcción, de-construcción y re-construcción de alternativas para debilitar y eliminar las causas o factores generadores de violencia (s) y, por ende, obstáculos para el ejercicio, reconocimiento, defensa y preservación de los DDHH en la IE.

Para desarrollar esta propuesta se plantea la necesidad de construir Núcleos Temáticos y Problemáticos entendidos como "la estrategia curricular que integra un conjunto de problemas con conocimientos académicos y cotidianos afines, que posibilitan definir líneas de investigación en torno al objeto de transformación, construir estrategias metodológicas disciplinarias, interdisciplinarias y transdisciplinarias que garantizan la relación teoría - práctica y la participación comunitaria en el proceso de formación", (López: 2003).

La investigación, entendida como el resultado de un proceso serio, responsable, válido, pertinente, resultado del asombro sistemático, de la duda permanente, convertida en práctica pedagógica esencial ${ }^{42}$, permitirá que las diferentes formas de violencia(s) que se presentan en la IE sean asumidas como Objetos de Investigación.

En la gráfica siguiente (Gráfica No. 1) se presenta de manera integrada la Propuesta Curricular Alternativa para garantizar el ejercicio, respeto, defensa y preservación de los $\mathrm{DDHH}$ en la IE, que pretende debilitar la

\footnotetext{
42 Importante señalar que en Colombia el proyecto nacional ONDAS que desarrolla la formación de niños, niñas y jóvenes investigadores se convierte en un referente concreto en la incorporación de la investigación como la práctica pedagógica esencial. Ver Colciencias-ONDAS
} 
concepción hegemónica que desliga la dinámica escolar del contexto socioeconómico y cultural del cual hace parte. Es conveniente señalar que "nuestra educación nos enseña a vivir de forma muy parcial e insuficiente, y es que nuestra educación se aparta de la vida, ignorando los problemas permanentes del vivir y dividiendo los conocimientos en compartimentos estancados. La tendencia tecno-económica, cada vez más poderosa e influyente, tiende a reducir la educación a la adquisición de competencias socio-profesionales en detrimento de las competencias existenciales, que pueden regenerar la cultura e introducir temas vitales en la enseñanza"(Morín: 2016).

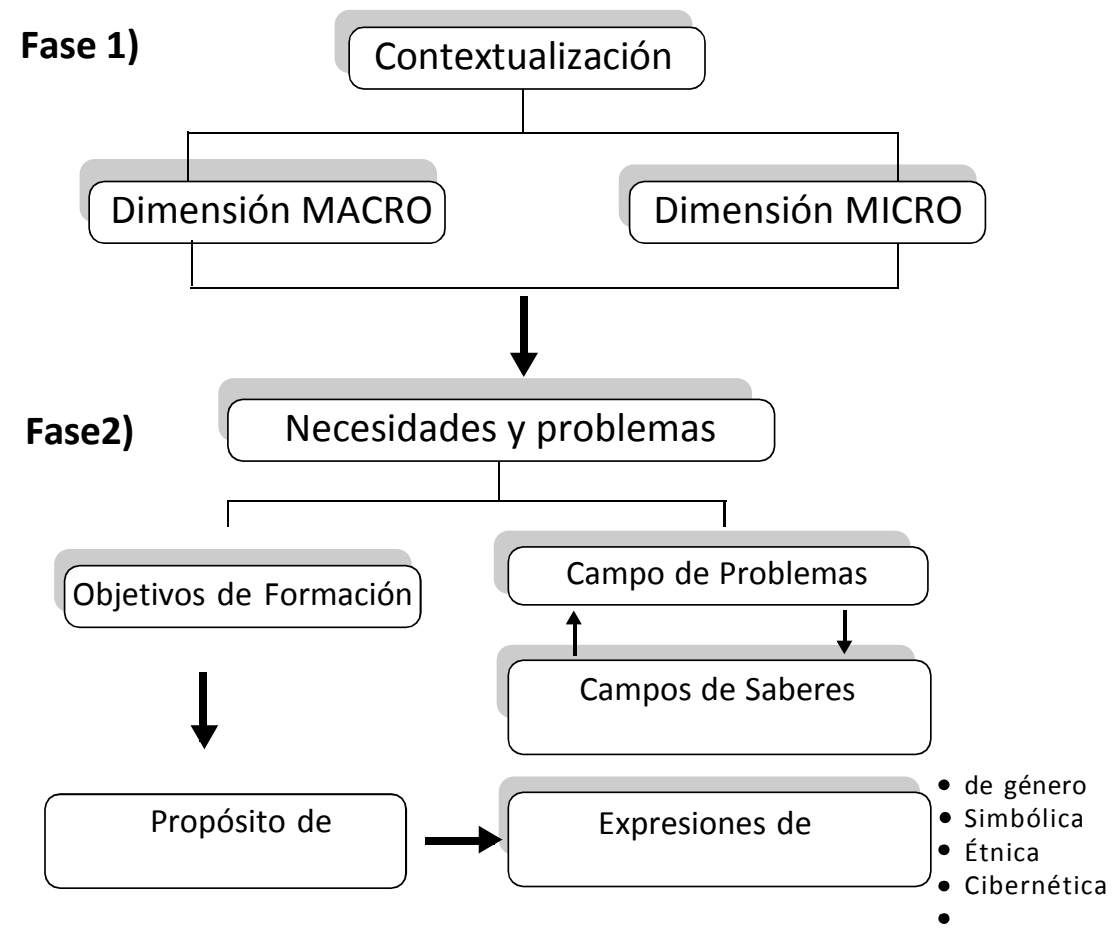

\section{Fase3)}

Enfoque Pedagógico de indagación Sistemática
Objetivos de Intervención

Proyectos curriculares específicos

Construcción de Núcleos Temátios y

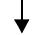

Problemáticos (sustentado en Investigación)

1

Desarrollos de seguimiento y evaluación 


\section{Criterios de Implementación de la Propuesta Curricular Alternativa}

Se plantea que en cada IE se cree un Comité Curricular integrado por representantes de los estudiantes, profesores, coordinadores, padres de familia y directivas que garanticen el desarrollo efectivo de lo propuesto y tengan como referente central de evaluación el debilitamiento y disminución de las causas y factores que dan lugar a que se presenten formas de violencia(s). Este Comité Curricular tendrá como una función esencial garantizar el ejercicio, defensa, reconocimiento y preservación de los $\mathrm{DDHH}$ en la IE. Además, sistematizará ${ }^{43}$ todos los proyectos, acciones de intervención, eventos, discusiones, participaciones y resultados obtenidos en el proceso de debilitar y erradicar las diferentes formas de violencia(s) en la IE.

\section{Acciones de seguimiento y acompañamiento}

Se hace necesario que el desarrollo de la Propuesta Curricular Alternativa incorpore nuevas formas de participación de las diferentes instituciones, organizaciones civiles, deportivas, culturales, a partir de la organización, desarrollo y evaluación de foros, debates, publicaciones, mesas de trabajo, grupos focales de cara a las problemáticas intervenidas que persigan en la práctica plantear un paradigma alternativo que advierta y argumente que lo "formativo no se agota en lo escolar" enseñar a vivir no es solo enseñar a leer, escribir y contar, no es solo enseñar los conocimientos básicos de historia, geografía, ciencias sociales y ciencias naturales. No es concentrarse en los saberes cuantitativos ni dar prioridad a las formaciones profesionales especializadas, sino introducir una cultura de base que incluya el conocimiento del conocimiento" (Morín: 2016).

Importante resaltar que la intención de esta investigación, además de responder por el logro de los objetivos propuestos, es invitar a una reflexión y acción sistemática de toda la sociedad que interviene en el proceso formativo de nuestros niños, niñas y jóvenes colombianos a transformar la actual realidad educativa que en el marco de un ideal de paz, convivencia, equidad, reconciliación nos permita acercarnos a la construcción de una

${ }^{43}$ La Sistematización se entiende como "la producción de conocimiento a partir de la experiencia". Mejía 2007 
respuesta válida, legítima y viable de cara a "(...) la suposición de que la raíz de nuestros males está en la crisis educativa y, asociada a esta, de que la mejora en la calidad de la educación permitiría superar nuestros históricos tormentos, parecería sustentarse en la aspiración de que es posible y deseable que alguna vez nos encontremos todos (sin distinciones de clases, razas, género o religión) en una sociedad en la que el bienestar y la equidad se hayan generalizado, en la que todos vivan alegres y felices, gozando de los beneficios que brinda el conocimiento cuando está a la altura de los desafíos que le plantean los nuevos tiempos" (Gentili:2015).

\section{Referentes bib liográficos}

- Alvarado, S; Vommaro, P. (2010). Jóvenes, cultura y política en América Latina: Algunos trayectos de sus relaciones, experiencias y lecturas (1960-2000). Buenos aires, Argentina: Homo Sapiens Ediciones.

- Alvarado, S; Vommaro, P. (2014). En busca de las condiciones juveniles latinoamericanas. Buenos Aires, Argentina: Centro Editorial CINDE.

- Bonilla Baquero, C. (2003). El des-encanto de la enseñanza: "Mamera y bacanidad". Neiva, Colombia: Editorial Universidad Surcolombiana.

- Díaz, V, Mario; López,J. Nelson. (2005) El Discurso Pedagógico Oficial y la Educación Superior en Colombia. Neiva, Huila: Colciencias, Editorial Universidad Surcolombiana.

- Gentili, P. (2012). Pedagogía de la igualdad. Buenos Aires, Argentina: Siglo Veintiuno editores.

- Jiménez, A; Infante, R. (2008). Infancia y ciudad en Bogotá: Una mirada desde las narrativas populares urbanas. Bogotá, Colombia: Fondo de Publicaciones Universidad Distrital Francisco José de Caldas.

- Kurlansky, M. (2015).No violencia: 25 lecciones sobre una idea peligrosa. Bogotá, Colombia: Penguin Random House Grupo Editorial

- Lomas, C. (1999); ¿lguales o diferentes? Género, diferencia sexual, lenguaje y educación. Buenos Aires, Argentina: Editorial Paidós

- López, N. Amaya, G; Lesmes, M; Malagón, A; Murillo, N (1990); Evaluación del rendimiento académico en los programas a distancia en Colombia. Quindío, Colombia: Centro de Publicaciones Universidad del Quindío

- López, N. (1995); La Restructuración Curricular de la Educación Superior: Hacia la Integración del Saber. Santafé de Bogotá, D.C, Colombia: Editorial Presencia Ltda. 
- López, N. (1996); Modernización Curricular de las Instituciones Educativas los PEI de cara al Siglo XXI. Santafé de Bogotá, D.C, Colombia: Editorial Libros \& Libres s.a.

- López, N. (1999); Tendencias actuales del desarrollo curricular en Colombia. Medellín, Colombia: Editorial Marín Vieco Ltda.

- López, N. (2002); Retos para la construcción curricular: De la certeza al paradigma de la incertidumbre creativa. Bogotá, Colombia: Cooperativa Editorial Magisterio

- López, N. (2004); Modelos y Prácticas Pedagógicas en la Educación Superior. Bogotá D.C. Colombia: Editora Guadalupe Ltda.

- López, N. (2006); Evaluación por Competencias: Un reto por enfrentar y un proceso por construir. Neiva, Huila, Colombia: Editorial Universidad Surcolombiana.

- López, N. (2007); Estado del Arte: Evaluación de la Calidad de la Educación en Colombia. Neiva, Huila, Colombia: Editorial Universidad Surcolombiana.

- López, N; Puentes, A; Guevara, L; Quiza, J. (2009); El Campo Escolar como Escenario de Cambio y Transformación. Neiva, Huila, Colombia: Editorial Universidad Surcolombiana.

- López, N. (2010); Seminario de Sistematización de Experiencias Investigativas en Educación. Neiva, Huila, Colombia: Editorial Universidad Surcolombiana.

- López, N; Puentes, A. (2011); Modernización Curricular de la Universidad Surcolombiana: Una experiencia de Integración e interdisciplinariedad. Neiva, Huila, Colombia: Editorial Universidad Surcolombiana.

- López, N; Puentes, A. (2011); Evaluación de la Calidad de la Educación en Colombia: Estado del Arte. Neiva, Huila, Colombia: Editorial Universidad Surcolombiana.

- López, N. (2013); Universidad y Estilos de Aprendizaje. Neiva, Huila, Colombia: Editorial Universidad Surcolombiana.

- López, N. s.f.; Factores de Retención Estudiantil en la Educación Superior en el Departamento del Huila. Neiva, Huila, Colombia: Editorial Universidad Surcolombiana.

- López, N. s.f.; Seminario Permanente sobre la Problemática Pedagógica en la Educación Superior. Neiva, Huila, Colombia: Editorial Universidad Surcolombiana. 
- Magendzo, A. (2009); Pensamiento e ideas - fuerza de la educación en derechos humanos en Iberoamérica. Santiago de Chile, Chile: Ediciones SM.

- Magendzo Kolstrein, A; Toledo Jofré, M; Rosenfeld Sekulovic, C. (2004). Intimidaciones entre estudiantes: Cómo identificarlos y cómo atenderlos. Santiago de Chile, Chile: Editorial LOM.

- Molina Andrade, A. (2013). Miradas contemporáneas en educación. Bogotá, Colombia: Universidad Distrital Francisco José de Caldas.

- Molina, N; Céspedes, M; Torres, E; Moreno, E; Sánchez, Z. (2015). Lenguaje, cultura e investigación: Problemas emergentes en educación. Bogotá, Colombia: Universidad Distrital Francisco José de Caldas.

- Mora Penagos, W. (2015). Educación en ciencias: Experiencias investigativas en el contexto de la didáctica, la historia, la filosofía y la cultura. Bogotá, Colombia: Universidad Distrital Francisco José de Caldas.

- Morales Nieto, J. (2015). ¿Qué es el Post Conflicto? Colombia después de la guerra. Bogotá, Colombia: Ediciones B Colombia S.A.

- Nussbaum, M. (2005). El cultivo de la humanidad: Una defensa clásica de la reforma en la educación liberal. Barcelona, España: Paidós.

- Paca. (2009) Revista PACA: Órgano de Difusión Investigativo. Neiva, Huila, Colombia; Editorial Universidad Surcolombiana.

- Paca. (2010) Revista PACA 2: Órgano de Difusión Investigativo. Neiva, Huila, Colombia; Editorial Universidad Surcolombiana.

- Paca. (2012) Revista PACA 3: Órgano de Difusión Investigativo. Neiva, Huila, Colombia; Editorial Universidad Surcolombiana.

- Paca. (2013) Revista PACA 4: Órgano de Difusión Investigativo. Neiva, Huila, Colombia; Editorial Universidad Surcolombiana.

- Paca (2013) Revista PACA 5: Órgano de Difusión Investigativo. Neiva, Huila, Colombia; Editorial Universidad Surcolombiana.

- Paca (2014) Revista PACA 6: Órgano de Difusión Investigativo. Neiva, Huila, Colombia; Editorial Universidad Surcolombiana.

- Paca (2015) Revista PACA 7: Órgano de Difusión Investigativo. Neiva, Huila, Colombia; Editorial Universidad Surcolombiana.

- Pereyra, B; Vommaro, P. (2011). (2a ed). Movimientos sociales y derechos humanos en la Argentina. Buenos Aires, Argentina: Ediciones CICCUS.

- Posada Carbó, E; Deas, M; Powell, Ch. (2014). La paz y sus principios. Bogotá, Colombia: Alfaomega Colombiana S.A.

- Rawls, J. (1997). El derecho de gentes y "Una revisión de la idea de razón pública". Madrid, España: Editorial Paidós. 
- Rengifo, A; Arias, D; Martínez, N; Castañeda, G; Velásquez, L; Gil, D et al. (2015). Escuela y educación superior: Temas para la reflexión. Bogotá, Colombia: Universidad Distrital Francisco José de Caldas.

- Revista del programa de Psicología de la Universidad Simón Bolívar. (2010). PsicoGente. Barranquilla, Colombia: Universidad Simón Bolívar.

- Santamaría Valero, F. (2008). Saberes y lenguajes: Una mirada interdisciplinar hacia los niños y los jóvenes. Bogotá, Colombia: Fondo de Publicaciones Universidad Distrital Francisco José de Caldas.

- Santamaría Valero, F. (2010). Diálogos con jóvenes: Escrituras y lecturas, violencias, sexualidad y rumba. Bogotá, Colombia: Universidad Distrital Francisco José de Caldas.

- Tapias, L; Orofino, M. (2012). Las escuelas como territorios de paz: Construcción social del niño y la niña como sujetos políticos en contextos del conflicto armado. Buenos Aires, Argentina: CLACSO.

- Valero, C; Rebellón, G; Sánchez, J. (2009). Escuela de Facilitadores Sociales: Hacia la construcción de una pedagogía de los vínculos. Cali, Colombia: Universidad Autónoma de Occidente.

- Valenzuela Arce, J. (2009). El futuro ya fue: socioantropología de I@s jóvenes en la modernidad. Ciudad de México, México: Casa Juan Pablos.

- Vallejo, C. (2012). Podemos cambiar el mundo. Ciudad de México, México: Ocen Sur. 